\title{
Phase Separation of Viscous Ternary Liquid Mixtures
}

\author{
Jang Min Park ${ }^{\mathrm{a}, \mathrm{b}}$, Roberto Mauri ${ }^{\mathrm{c}}$, Patrick D. Anderson ${ }^{\mathrm{a}, *}$ \\ ${ }^{a}$ Department of Mechanical Engineering, Eindhoven University of Technology, 5600 MB \\ Eindhoven, The Netherlands. \\ ${ }^{b}$ Institute for Complex Molecular Systems, Eindhoven University of Technology, 5600 \\ $M B$ Eindhoven, The Netherlands. \\ ${ }^{c}$ Department of Chemical Engineering, Industrial Chemistry and Material Science, \\ University of Pisa, 56126 Pisa, Italy.
}

\begin{abstract}
In this work we study the demixing of ternary liquid mixtures. Our theoretical model follows the standard diffuse interface model, where convection and diffusion are coupled via a body force, expressing the tendency of the mixture to minimize its free energy. This driving force induces a material flux which, in most cases, is much larger than that due to pure molecular diffusion. Here we model the behavior of a very viscous polymer melt, so that the Peclet number, expressing the ratio between convective and diffusive mass fluxes, is small. Two examples are presented, describing the phase separation of ternary mixtures in two and three phases, respectively, following an initial quench to an unstable state of their phase diagram. In the first case, as expected, we see that the growth of the domain size follows the well known diffusion-driven scaling, $R(t) \propto t^{1 / 3}$. On the other hand, in the second example, the domain size growth follows the usual $t^{1 / 3}$ scaling until the symmetry among the three phases breaks down and the domain size of
\end{abstract}

\footnotetext{
*Corresponding author, email: P.D.Anderson@tue.nl
} 
two of the three phases decrease sharply. After that point, the morphology of the system becomes more regular, almost crystal-like, and the three phases start to grow again, with the same growth rate $R(t) \propto t^{n}$, with $n=0.11$. Keywords: Phase Separation, Diffuse Interface Model, Ternary Liquid Mixture

\section{Introduction}

The diffuse interface model was developed originally to describe nearcritical behavior of single-component fluids and partially miscible binary mixtures (Cahn and Hilliard, 1958, 1959; Hohenberg and Halperin, 1977; Lowengrub and Truskinovsky, 1998; Vladimirova et al., 1999), and it has been widely used to study many kinds of physical phenomena of binary mixtures such as mixing of viscous liquids (Vladimirova and Mauri, 2004), droplet dynamics (Yue et al., 2004) and structure development of polymer blends (Prusty et al., 2007; Keestra et al., 2011).

Despite many industrial and biochemical processes involve mixtures with three or more components, only a few theoretical and numerical works have studied these systems. In particular, Huang et al. (1995) analyzed numerically the dynamics of phase separation of ternary alloys (i.e. where convective effects can be neglected) into two and three phases by solving the nonlinear spinodal decomposition equations in two dimensions. Examining the dynamical scaling and the growth laws for the late stages of separation, they saw that the growth law $R(t) \propto t^{1 / 3}$ is always obeyed, despite the fact that the self-similar regime is achieved very slowly in ternary systems. Later, Kim et al. (2004); Kim and Lowengrub (2005) developed a full 
Navier-Stokes/Cahn-Hilliard code to model the phase mixing/demixing and the Rayleigh instability of ternary mixtures using a diffuse interface model in the low Reynolds number regime. There, applying boundary integral methods, the effects of surfactants on drop dynamics, tip-streaming and drop deformation have been investigated. Phase-field ternary mixture models have also been of interest for modeling many different physical phenomena, for exmaple, solidification and microstructure evolution in ternary alloy systems (Kobayashi et al., 2003), mutual diffusion effects in partially miscible polymer blends (Tufano et al., 2010), and surfactant-induced emulsion coarsening (Lamorgese and Banerjee, 2011).

In this work, starting from the already existent results for two component systems (Lamorgese and Mauri, 2005), we develop a general model of ternary mixtures, in which the Navier-Stokes equation is coupled to generalized Cahn-Hilliard equations for the phase variables. Compared to previous models, the present one has the advantage of simplicity and thermodynamic consistency, without employing any ad hoc term that cannot be directly related to macroscopic, easily measured parameters.

\section{The governing equations}

\subsection{Multi-component mixtures at equilibrium}

Consider a homogeneous mixture of $N$ species $A_{k}(k=1,2 \ldots N)$, with molar fractions $x_{k}$, kept at temperature $T$ and pressure $P$. For sake of simplicity, in our model we assume that the molecular weights, specific volumes and viscosities of all species are the same, namely $M_{k}=M_{w}, \bar{V}_{k}=\bar{V}$ and $\eta_{k}=\eta$, for all species $k$, so that molar, volumetric and mass fractions are all 
equal to each other, and the mixture viscosity is composition-independent. The equilibrium state of this system is described by the "coarse-grained" free energy functional, that is the molar Gibbs energy of mixing, $\Delta g^{t h}$,

$$
\Delta g^{t h}=g^{t h}-\sum_{k=1}^{N} g_{k} x_{k},
$$

where $g^{t h}$ is the energy of the mixture at equilibrium, while $g_{k}$ is the molar free energy of pure species $A_{k}$ at temperature $T$ and pressure $P$. The free energy $\Delta g^{t h}$ is the sum of an ideal part $\Delta g^{i d}$ and a so-called excess part $g^{e x}$, with

$$
\Delta g^{i d}=R T \sum_{k=1}^{N} x_{k} \log x_{k},
$$

where $R$ is the gas constant, while the excess molar free energy can be expressed as,

$$
g^{e x}=\frac{1}{2} R T \sum_{i, k=1}^{N} \Psi_{i k} x_{i} x_{k},
$$

where $\Psi_{i k}$ are functions of $T$ and $P$, with $\Psi_{i k}=\Psi_{k i}$ and $\Psi_{i i}=0$. This expression can be generally derived by considering the molecular interactions between nearest neighbors or summing all pairwise interactions throughout the whole system (Lifshitz and Pitaevskii, 1984). As shown by Mauri et al. (1996), Eq. (3) it can also be derived from first principles, assuming that the pairwise forces between identical molecules, $F_{i, i}$ are all equal to each other and larger than the pairwise forces among unequal molecules, $F_{i, j}$ (with $i \neq j$ ), i.e. $F_{i, i}=F_{j, j}>F_{i, j}$, obtaining an expression for $\Psi_{i j}$ which depends on $\left(F_{i, i}-F_{i, j}\right)$. In the following, we shall assume that $P$ is fixed, so that the physical state of the mixture at equilibrium depends only on $T$ and $x_{i}$.

Now, it is well-known that any variation of the molar free energy can be 
written as (Prausnitz et al., 1986),

$$
d g^{t h}=R T \sum_{i=1}^{N} \mu_{i}^{t h} d x_{i}
$$

where $\mu_{i}^{t h}$ denotes the chemical potential of species $A_{i}$ in solution, i.e.,

$$
\mu_{i}^{t h}=\frac{1}{R T} \frac{\partial\left(c g^{t h}\right)}{\partial c_{i}}
$$

with $c_{i}=c x_{i}$ denoting the mole densities, that is the number of moles per unit volume, of species $A_{i}$, and $c=\sum c_{i}$ is the total mole density. In our case we obtain:

$$
\mu_{i}^{t h}=\frac{g^{t h}}{R T}+\log x_{i}+\sum_{k=1}^{N} \Psi_{i k}\left(1-x_{i}\right) x_{k}-\sum_{j, k \neq i=1}^{N} \Psi_{j k} x_{j} x_{k} .
$$

Since free energy is an extensive quantity, it is easy to show that chemical potentials represent the amount of free energy due to each species, i.e.

$$
g^{t h}=R T \sum_{i=1}^{N} \mu_{i}^{t h} x_{i}
$$

Therefore, comparing Eqs. (4) and (7), we obtain the Gibbs-Duhem relation,

$$
\sum_{i=1}^{N} x_{i} d \mu_{i}^{t h}=0
$$

Considering that $\sum_{i=1}^{N} x_{i}=1$, we see that Eq. (4) can be rewritten as:

$$
d g^{t h}=R T \sum_{i=1}^{N-1} \mu_{i N}^{t h} d x_{i}
$$

where $\mu_{i j}^{t h} \equiv \mu_{i}^{t h}-\mu_{j}^{t h}$. Accordingly, we see that the quantities $x_{i}$ and $R T \mu_{i N}^{t h}$ are thermodynamically conjugated, i.e. $R T \mu_{i N}^{t h}=\partial g_{e q} / \partial x_{i}$. In fact, applying this expression, we obtain:

$$
\mu_{i j}^{t h}=\ln \frac{x_{i}}{x_{j}}+\Psi_{i j}\left(x_{j}-x_{i}\right)+\sum_{k \neq i, j=1}^{N}\left(\Psi_{i k}-\Psi_{j k}\right) x_{k},
$$


where, by definition, $\mu_{i j}^{t h} \equiv \mu_{i k}^{t h}-\mu_{j k}^{t h}$ and $\mu_{i j}^{t h}=-\mu_{j i}^{t h}$. The same result could be obtained directly from Eq. (6).

\subsection{Non-local terms}

In order to take into account the effects of spatial inhomogeneities, following Cahn and Hilliard $(1958,1959)$, we assume that the total, or generalized, free energy $\widetilde{g}$ is the sum of an equilibrium part and a non-local part,

$$
\widetilde{g}=g^{t h}+g^{n l}
$$

where the latter is given by the following expression:

$$
g^{n l}=\frac{1}{4} R T a^{2} \sum_{i=1}^{N}\left(\nabla x_{i}\right)^{2}
$$

Here $a$ represents the typical length of spatial inhomogeneities in the composition which, as shown by van der Waals (1894), is proportional to the surface tension between the two phases. Note that, considering that $\sum_{i=1}^{N} x_{i}=1$, this expression can also be written as:

$$
g^{n l}=-\frac{1}{2} R T a^{2} \sum_{i \neq j=1}^{N} \nabla x_{i} \nabla x_{j}=\frac{1}{2} R T a^{2} \sum_{i=1}^{N-1}\left(\nabla x_{i} \sum_{j \geq i}^{N-1} \nabla x_{j}\right) .
$$

Now, chemical potentials can be generalized as follows:

$$
\widetilde{\mu}_{i}=\frac{1}{R T} \frac{\delta(c \widetilde{g})}{\delta c_{i}}=\mu_{i}^{t h}+\mu_{i}^{n l}
$$

where $\mu_{i}^{\text {th }}$ is defined in Eq. (5), while,

$$
\mu_{i}^{n l}=-\frac{1}{R T} \nabla \cdot\left(\frac{\partial\left(c g^{n l}\right)}{\partial \nabla c_{i}}\right)
$$


Consequently we obtain:

$$
\mu_{i}^{n l}=\frac{a^{2}}{2} \nabla \cdot\left[-\left(1-x_{i}\right) \nabla x_{i}+\sum_{j \neq i=1}^{N} x_{j} \nabla x_{j}\right] .
$$

It can be shown that, being $g^{n l}$ a quadratic function of $\nabla x_{i}$, we obtain:

$$
2 g^{n l}=\sum_{i=1}^{N} \mu_{i}^{n l} x_{i}
$$

In addition,

$$
-\nabla g^{n l}=\sum_{i=1}^{N} \mu_{i}^{n l} \nabla x_{i}
$$

Consequently,

$$
\sum_{i=1}^{N} x_{i} \nabla \mu_{i}^{n l} \neq 0
$$

showing that the Gibbs-Duhem relation cannot be extended to the non local part of the free energy.

Finally, since we are mostly interested in the chemical potential differences, we have:

$$
\mu_{i j}^{n l}=-\frac{a^{2}}{2} \nabla^{2}\left(x_{i}-x_{j}\right)
$$

In particular, for ternary mixtures, we obtain:

$$
\begin{aligned}
& \widetilde{\mu}_{12}=\ln \frac{x_{1}}{x_{2}}+\Psi_{12}\left(x_{2}-x_{1}\right)+\left(\Psi_{13}-\Psi_{23}\right) x_{3}-\frac{a^{2}}{2} \nabla^{2}\left(x_{1}-x_{2}\right) \\
& \widetilde{\mu}_{23}=\ln \frac{x_{2}}{x_{3}}+\Psi_{23}\left(x_{3}-x_{2}\right)+\left(\Psi_{21}-\Psi_{31}\right) x_{1}-\frac{a^{2}}{2} \nabla^{2}\left(x_{2}-x_{3}\right) \\
& \widetilde{\mu}_{31}=\ln \frac{x_{3}}{x_{1}}+\Psi_{31}\left(x_{1}-x_{3}\right)+\left(\Psi_{32}-\Psi_{12}\right) x_{2}-\frac{a^{2}}{2} \nabla^{2}\left(x_{3}-x_{1}\right) .
\end{aligned}
$$

Note that, by definition, $\widetilde{\mu}_{12} \equiv \widetilde{\mu}_{13}+\widetilde{\mu}_{32}$ and $\widetilde{\mu}_{i j}=-\widetilde{\mu}_{j i}$. 


\subsection{The equations of motion}

Consider a mixture of $N$ components, where the $k$-th species has density $\rho_{k}$, volume fraction $\phi_{k}$ and velocity $\mathbf{v}_{k}$. The mass balance equation for each component can be written as:

$$
\frac{\partial\left(\rho_{k} \phi_{k}\right)}{\partial t}+\nabla \cdot\left(\rho_{k} \phi_{k} \mathbf{v}_{k}\right)=0
$$

Now, define the mixture density and the mixture velocity as the following mass-average quantities,

$$
\rho=\sum_{k=1}^{N} \rho_{k} \phi_{k}
$$

and

$$
\mathbf{v}=\frac{1}{\rho} \sum_{k=1}^{N} \phi_{k} \rho_{k} \mathbf{v}_{k}=\sum_{k=1}^{N} c_{k} \mathbf{v}_{k}
$$

where $c_{k}=\phi_{k} \rho_{k} / \rho$ is the mass fraction of the $k$-th component. Summing equation (24) for all k's, we trivially obtain the continuity equation,

$$
\frac{\partial \rho}{\partial t}+\nabla \cdot(\rho \mathbf{v})=0
$$

In the following, we assume that all components have the same density $\rho$ and molecular weight $M_{w}$, so that $c_{k}=\phi_{k}=x_{k}$ represent the mass, volume and molar fractions of the $k$-th component. Accordingly, the mixture is incompressible, i.e. Eq. (27) reduces to

$$
\nabla \cdot \mathbf{v}=0
$$

while the mass concentration equations becomes:

$$
\dot{x}_{k}=\frac{\partial x_{k}}{\partial t}+\mathbf{v} \cdot \nabla x_{k}=-\nabla \cdot \mathbf{J}_{k}
$$


where the dot indicates the advection derivative with respect to the mixture velocity $\mathbf{v}$ and

$$
\mathbf{J}_{k}=x_{k}\left(\mathbf{v}_{k}-\mathbf{v}\right)
$$

is the volumetric diffusive flux, depending on the the velocity of the $k$-th component with respect to the mean, with $\sum \mathbf{J}_{k}=\mathbf{0}$. Obviously, since $\sum c_{k}=1$, only $N-1$ of these equations are independent.

Equations (28) and (29) must be couple with the Navier-Stokes equation,

$$
\rho \dot{\mathbf{v}}+\nabla p=\nabla \cdot \boldsymbol{\tau}+\mathbf{f}_{\phi},
$$

where $\boldsymbol{\tau}$ is the viscous stress tensor, while $\mathbf{f}_{\phi}$ is the Korteweg reversible force,

$$
\mathbf{f}_{\phi}=\frac{\rho R T}{M_{w}} \frac{\delta \widetilde{g}}{\delta \mathbf{r}}=\frac{\rho R T}{M_{w}} \sum_{i=1}^{N}\left(\widetilde{\mu}_{i} \nabla x_{i}\right) .
$$

Note that, since $\widetilde{\mu}_{i}=\mu_{i}^{t h}+\mu_{i}^{n l}$ and considering that

$$
\sum \mu_{i}^{t h} \nabla x_{i}=\nabla g^{t h}
$$

after redefining the pressure as $p-\rho R T g^{\text {th }}$, the above equation can be rewritten replacing $\widetilde{\mu}_{i}$ with $\mu_{i}^{n l}$. Now, considering that $\sum x_{i}=1$, we finally obtain:

$$
\mathbf{f}_{\phi}=\frac{\rho R T}{M_{w}} \sum_{i=1}^{N-1}\left(\mu_{i N}^{n l} \nabla x_{i}\right) .
$$

\subsection{The constitutive equations for the diffusive fluxes}

Here, for sake of simplicity, we assume that the viscosities of all components are the same, so that the viscous stress in Eq. (31) can be written:

$$
\boldsymbol{\tau}=\eta\left(\nabla \mathbf{v}+(\nabla \mathbf{v})^{\dagger}\right)
$$

where $\eta$ is the uniform viscosity of the mixture. 
As for the material diffusive fluxes, generalizing the two component case, we use the following constitutive relations,

$$
\mathbf{J}_{i}=-\sum_{j=1}^{N} D_{i j} x_{i} x_{j} \nabla \widetilde{\mu}_{i j}
$$

here $D_{i j}=D_{j i}$ and $\mu_{i j}=\mu_{i}-\mu_{j}$, so that $\sum \mathbf{J}_{i}=\mathbf{0}$ identically, as it should.

Note that, had we assumed the following constitutive relation,

$$
\mathbf{J}_{i}=-D x_{i} \nabla \mu_{i},
$$

the sum of the diffusive fluxes $\mathbf{J}_{i}$ would not be zero, as the Gibbs-Duhem relation is not satisfied by the non-local part of the chemical potential.

In particular, for ternary mixtures, assuming that the diffusion coefficients are all equal, we obtain:

$$
\begin{aligned}
& \mathbf{J}_{1}=-D x_{1} x_{2} \nabla \widetilde{\mu}_{23}-D x_{1}\left(1-x_{1}\right) \nabla \widetilde{\mu}_{13}, \\
& \mathbf{J}_{2}=-D x_{1} x_{2} \nabla \widetilde{\mu}_{13}-D x_{2}\left(1-x_{2}\right) \nabla \widetilde{\mu}_{23} .
\end{aligned}
$$

Note that this makes the concentration equation a fourth-order nonlinear advection diffusion equation, which is a generalization of the classical CahnHilliard equation, used to describe the phase separation of binary mixtures.

The above equations can be re-scaled defining:

$$
\widehat{\mathbf{r}}=\frac{\mathbf{r}}{a} ; \quad \widehat{t}=\frac{t}{\left(a^{2} / D\right)} ; \quad \widehat{\mathbf{v}}=\frac{\mathbf{v}}{V} ; \quad \widehat{p}=\frac{p}{\eta V / a},
$$

where $V$ is a characteristic velocity, which can be estimated through Eqs. (31) as $V \approx f_{\phi} a^{2} / \eta$, with $f_{\phi} \approx \rho R T /\left(a M_{w}\right)$. At the end, we obtain the following non-dimensional system of equations, which is the ternary version of the model $H$, in the nomenclature of Hohenberg and Halperin (1977), 


$$
\begin{gathered}
N_{S c}^{-1} \frac{\partial \mathbf{v}}{\partial t}+N_{R e} \mathbf{v} \cdot \nabla \mathbf{v}=-\nabla p+\nabla^{2} \mathbf{v}+\left(\widetilde{\mu}_{13} \nabla x_{1}+\widetilde{\mu}_{23} \nabla x_{2}\right) ; \\
\nabla \cdot \mathbf{v}=0 \\
\frac{\partial x_{1}}{\partial t}+N_{P e} \mathbf{v} \cdot \nabla x_{1}=\nabla \cdot\left[x_{1} x_{2} \nabla \widetilde{\mu}_{23}+x_{1}\left(1-x_{1}\right) \nabla \widetilde{\mu}_{13}\right] \\
\frac{\partial x_{2}}{\partial t}+N_{P e} \mathbf{v} \cdot \nabla x_{2}=\nabla \cdot\left[x_{1} x_{2} \nabla \widetilde{\mu}_{13}+x_{2}\left(1-x_{2}\right) \nabla \widetilde{\mu}_{23}\right]
\end{gathered}
$$

where hats have been omitted for simplicity, while $\widetilde{\mu}_{13}$ and $\widetilde{\mu}_{23}$ are given by Eq. (22) and (23). Here, $N_{R e}=V a / \nu$ is the Reynolds number, while $N_{P e}=$ $V a / D=N_{R e} N_{S c}$ defines the capillary Peclet number, with $N_{S c}=\nu / D$ denoting the Schmidt number. Here, we will consider the case where $N_{P e}=$ 0.05 and $N_{S c}=10^{11}$, corresponding to a typical value of polymer melts, where $a \approx 10^{-5} \mathrm{~cm}, D \approx 10^{-9} \mathrm{~cm}^{2} / \mathrm{s}, \nu \approx 10^{2} \mathrm{~cm}^{2} / \mathrm{s}, M_{w} \approx 10^{4} \mathrm{~g} / \mathrm{gmole}$. Clearly, in this case, Eq. (41) reduces to a creeping flow equation, as its LHS can be neglected.

\section{Numerical results}

Physically, we consider an instantaneous quench bringing the mixture from its single-phase, stable and homogeneous initial state to an unstable final state, corresponding to a point in its phase diagram lying within the coexistence curve.

Two types of ternary mixtures were considered, corresponding to mixtures that phase separate in two and in three coexisting phases. In the following, these two cases will be treated separately. 


\subsection{Numerical methods}

The two second-order differential equations representing the chemical potential and the concentration equation are solved in a coupled way for both phases. For the temporal discretization a first-order Euler implicit scheme was employed, with a $4 \times 10^{-3}$ non-dimensional time step. The nonlinear terms in the chemical potential equation and momentum balance equation were linearized by a standard Picard method. A second-order finite element method was used for spatial discretization of the set of equations, using a square periodic domain, with $40 \times 40$ elements, assuming bi-periodic boundary condition.

Details about the iteration scheme can be found in Keestra et al. (2003) and Khatavkar et al. (2006). The flow problem was solved using the velocitypressure formulation and discretized by a standard Garlekin finite element method. Taylor-Hood quadrilateral elements with continuous pressure, that employ a biquadratic approximation for the velocity and a bilinear approximation for the pressure, are used. The resulting discretized second-order linear algebraic equation was solved using a direct method based on a sparse multifrontal variant of Gaussian elimination (HSL/MA41). (Amestoy and Duff, 1989; Amestoy and Puglisi, 2002)

The average domain size is calculated using a correlation function defined as

$$
G(\mathbf{R}, t)=\frac{1}{N_{n}} \sum_{i=1}^{N_{n}}\left(x_{k}\left(\mathbf{r}_{i}+\mathbf{R}, t\right)-x_{\mathrm{av}}\right) \cdot\left(x_{k}\left(\mathbf{r}_{i}, t\right)-x_{\mathrm{av}}\right),
$$

where $N_{n}$ is total number of nodal points, $\mathbf{r}_{i}$ and $\mathbf{R}$ are the lattice vectors, and $x_{\mathrm{av}}$ is the average molar fraction of species $k$. Radial averaging is carried 
out using a Brillouin zone function to eliminate any directional effects

$$
g(R, t)=\frac{1}{N_{r}} \sum_{R-\Delta R / 2<|\mathbf{R}|<R+\Delta R / 2} G(\mathbf{R}, t),
$$

where $N_{r}$ is the number of nodal points used in the averaging. The first intersection of the correlation function with the $R$-axis is used as a measure for the domain size (Prusty et al., 2007).

\subsection{Two-phase mixture}

Assume that $\Psi_{12}=4$ and $\Psi_{13}=\Psi_{23}=0$, corresponding to the triangular phase diagram of Fig. 1. Here, the line $1-2$ represents an almost immiscible binary mixture, with equilibrium points having composition $\left(x_{1} ; x_{2}\right)=(x ; 1-$ $x$ ) and $(1-x ; x)$, with $x=0.021$ (we find that from Eq. (21), imposing that $\mu_{12}=0$, with $x_{1}$ and $x_{2}$ uniform, so that there is no contribution from the non-local part; the additional solution, $x=1 / 2$, is unstable.) As we add the 3-component, which is miscible with both 1 and 2, the mixture becomes more and more miscible, until we reach the critical point $C$ at $\left(x_{1}=x_{2}=0.224 ; x_{3}=0.552\right)$ (we find that imposing that at the critical point $x_{1}=x_{2}=x$, so that $x_{3}=1-2 x$, and then imposing that the chemical potential differences are all zero.) Note that because of symmetry all tie lines are parallel to $1-2$ axis.

Initially, the mixture is assumed to be homogeneous with $x_{1}=x_{2}=0.35$ and $x_{3}=0.3$, corresponding to a state well inside the unstable region of the phase diagram. When a small random perturbation is superimposed, as shown in Figs. 2-3, we see that the mixture separates into two phases, one rich of component 1 and the other rich of component 2, while component 3 , being equally miscible within 1 and 2 , distributes homogeneously within the 
mixture. As one should expect, the dynamics of phase separation in this case is identical to that for binary mixtures and therefore all the comments that were made for binary mixtures can also be applied here.(Huang et al., 1995; Lamorgese and Mauri, 2008) In particular, the typical size $R$ of single phase domains grows with time as $R(t) \propto t^{n}$, where the power-law scaling exponent $n=1 / 3$ can be obtained imposing that the capillary force is balanced by the viscous force. The agreement shown in Fig. 3 can be considered as an indication that our theoretical model of ternary mixtures is sound and also as a validation of the numerical scheme.

\subsection{Three-phase mixture}

Consider a symmetric ternary mixture, $\Psi_{12}=\Psi_{13}=\Psi_{23}=\Psi=4$. As shown in Fig. 4, at the center of the triangular phase diagram there in a smaller triangle (called tie triangle), whose three vertices represent the composition of three coexisting phases, $\alpha$ in point $A, \beta$ in point $B$ and $\gamma$ in point $C$ (Huang et al., 1995). If $x$ is the distance between $A$ and the $1-2$ axis (and between $B$ and the $1-3$ axis as well as between $C$ and the $2-3$ axis, by symmetry), the compositions of the three coexisting phases are:

$$
\mathbf{x}^{\alpha}=(1-2 x, x, x) ; \quad \mathbf{x}^{\beta}=(x, 1-2 x, x) ; \quad \mathbf{x}^{\gamma}=(x, x, 1-2 x),
$$

with $x<1 / 3$. Then, from Eq. (21)-(23), we see that all thermodynamic chemical potential differences in $A, B$ and $C$ are identically zero, which gives us the following relation:

$$
\mu_{12}^{\alpha}=\ln \frac{1-2 x}{x}+\Psi(3 x-1)=0 .
$$

(all the other relations reduce to this one or are identically satisfied) So, assuming that we have three phases, knowing $\Psi$, we can find $x$, i.e. the 
composition of the three coexisting phases. Here, we see that the composition $x=1 / 3$ always satisfies this expression. However, this correspond to a point of stable equilibrium only for $\Psi<8 / 3$. Clearly, we see that when $\Psi=8 / 3$ the three points $A, B$ and $C$ converge at center of the triangle, while when $\Psi \gg 8 / 3$ they end up at the three vertices 1,2 and 3 of the phase diagram, as it should be. In our case, with $\Psi=4$, we find $x=0.023$, corresponding to $\mathbf{x}^{\alpha}=(0.954 ; 0.023 ; 0.023) ; \mathbf{x}^{\beta}=(0.023 ; 0.954 ; 0.023) ; \mathbf{x}^{\gamma}=$ $(0.023 ; 0.023 ; 0.954)$.

Initially, the mixture is assumed to be homogeneous with $x_{1}=x_{2}=$ $x_{3}=1 / 3$, corresponding again to an unstable state. When a small random perturbation is superimposed, as shown in Figs. 5-7, we see that the mixture separates into three coexisting phases which, initially, grow together, with the usual diffusion-driven $t^{1 / 3}$ growth law. Unexpectedly, though, at about $t=150$, the perfect symmetry among the three components breaks down, as phase $\gamma$ continues to separate quickly, as seen in Fig. 7, while the other two phases separate later, as their domains appear to shrink sharply (see Figs. 5d-e and Figs. 6d-e). In addition, the morphology of the system from this point on becomes more regular, almost crystal-like, as if the different phases were forced to orient themselves at certain angles with respect to each other. Perhaps because of that (but the reason of this behavior should be further investigated), after the symmetry break the domain size of all three phases grow following the same power law, $R(t) \propto t^{n}$, where $n=0.11$ (see Fig. 8). As this phenomenon has not been observed before, further investigation is required to confirm and better understand it. 


\section{Conclusions}

In this study we have presented phase separation of ternary liquid mixtures in a bi-periodic square domain. The theoretical model for three component system is developed by extending the existing two component model, where the Navier-Stokes equation is coupled with the Cahn-Hilliard equations of the phase variables. A second-order finite element method is used to discretize the set of equations, and the nonlinear terms are linearized by a standard Picard method.

We study two examples of the phase separation of ternary mixtures which are initially quenched to an unstable state of their phase diagram, assuming that the process is diffusion-driven, so that the Peclet number is small and the Reynolds number is negligibly small. In the first example, two components are almost immiscible with each other while the third component is equally miscible with the others, so that the system separates into two phases. In this case, after the initial quench, phase separation is driven by the two immiscible components, while the third component is distributed homogeneously within the mixture, so that the growth of the domain size follows predictably the well known diffusion-driven scaling, $R(t) \propto t^{1 / 3}$. In the second example, the ternary mixture is perfectly symmetric and consists of three components, which are mutually partially miscible. In this case, after the initial quench, the domain size growth follows the usual , $t^{1 / 3}$ scaling, until the symmetry among the three phases breaks down and the domain size of two of the three phases decrease sharply. After that point, the morphology of the system becomes more regular, almost crystal-like, and the three phases start to grow again, with the same growth rate $R(t) \propto t^{n}$, with $n=0.11$. 
As this result is new and quite unexpected, further studies are required to confirm it, providing also an understanding of this complex phenomenon.

\section{References}

Amestoy, P. R., Duff, I. S., 1989. Vectorization of a multiprocessor multifrontal code. Int. J. Supercomput. Appl. 3 (3), 41-59.

Amestoy, P. R., Puglisi, C., 2002. An unsymmetrized multifrontal LU factorization. SIAM J. Matrix Anal. Appl. 24 (2), 553-69.

Cahn, J. W., Hilliard, J. E., 1958. Free energy of a nonuniform system. I. Interfacial free energy. J. Chem. Phys. 28, 258-67.

Cahn, J. W., Hilliard, J. E., 1959. Free energy of a nonuniform system. III. Nucleation in a two-component incompressible fluid. J. Chem. Phys. 31, 688-99.

Hohenberg, P. C., Halperin, B. I., 1977. Theory of dynamic critical phenomena. Rev. Mod. Phys. 49, 435-79.

Huang, C., Olvera de la Cruz, M., Swift, B. W., 1995. Phase separation of ternary mixtures: Symmetric polymer blends. Macromolecules 28, 79968005 .

Keestra, B. J., Goossens, J. G. P., Anderson, P. D., 2011. Structure development of PMMA/SAN blends in shear flow. Chem. Eng. Sci. 66 (21), 4960-71. 
Keestra, B. J., Van Puyvelde, P. C. J., Anderson, P. D., Meijer, H. E. H., 2003. Diffuse interface modeling of the morphology and rheology of immiscible polymer blends. Phys. Fluids 15 (9), 2567-75.

Khatavkar, V. V., Anderson, P. D., Meijer, H. E. H., 2006. On scaling of diffuse-interface models. Chem. Eng. Sci. 61 (8), 2364-78.

Kim, J., Kang, K., Lowengrub, J., 2004. Conservative multigrid methods for ternary Cahn-Hilliard systems. Comm. Math. Sci. 2 (1), 53-77.

Kim, J., Lowengrub, J., 2005. Phase field modeling and simulation of threephase flows. Interfaces Free Boundaries 7 (4), 435-66.

Kobayashi, H., Ode, M., Kim, S. G., Kim, W. T., Suzuki, T., 2003. Phasefield model for solidification of ternary alloys coupled with thermodynamic database. Scr. Mater. 48 (6), 689-94.

Lamorgese, A., Banerjee, S., 2011. Insoluble surfactant effects on emulsion coarsening in a gravitational field via phase-field ternary mixture model. In: Proceedings of the $8^{\text {th }}$ SINTEF/NTNU Conference, Trondheim, Norway.

Lamorgese, A. G., Mauri, R., 2005. Nucleation and spinodal decomposition of liquid mixtures. Phys. Fluids 17, 034107.

Lamorgese, A. G., Mauri, R., 2008. Diffuse-interface modeling of phase segregation in liquid mixtures. Int. J. Multiphase Flow 34 (6), 987-95.

Lifshitz, E. M., Pitaevskii, L. P., 1984. Physical Kinetics. Pergamon Press, New York. 
Lowengrub, J., Truskinovsky, L., 1998. Quasi-incompressible Cahn-Hilliard fluids and topological transitions. Proc. Roy. Soc. A 454, 2617-54.

Mauri, R., Shinnar, R., Triantafyllou, G., 1996. Spinodal decomposition in binary mixtures. Phys. Rev. E 53, 2613-23.

Prausnitz, J. M., Lichtenthaler, R. N., de Azevedo, E. G., 1986. Molecular thermodynamics of fluid-phase equilibria, 2nd Edition. Prentice Hall.

Prusty, M., Keestra, B., Goossens, J., Anderson, P., 2007. Experimental and computational study on structure development of PMMA/SAN blends. Chem. Eng. Sci. 62 (6), 1825-37.

Tufano, C., Peters, G., Meijer, H., Anderson, P., 2010. Effects of partial miscibility on drop-wall and drop-drop interactions. J. Rheol. 54 (1), 15983.

van der Waals, J. D., 1894. The thermodynamic theory of capillarity under the hypothesis of a continuous variation of density. Z. Phys. Chem. Stöchiom. Verwandtschaftsl. 13, 657-725, translated and reprinted in J. Stat. Phys. 20, 200-44 (1979).

Vladimirova, N., Malagoli, A., Mauri, R., 1999. Two-dimensional model of phase segregation in liquid binary mixtures. Phys. Rev. E 60, 6968-77.

Vladimirova, N., Mauri, R., 2004. Mixing of viscous liquid mixtures. Chem. Eng. Sci. 59, 2065-9.

Yue, P., Feng, J. J., Liu, C., Shen, J., 2004. A diffuse-interface method for simulating two-phase flows of complex fluids. J. Fluid Mech. 515, 293-317. 


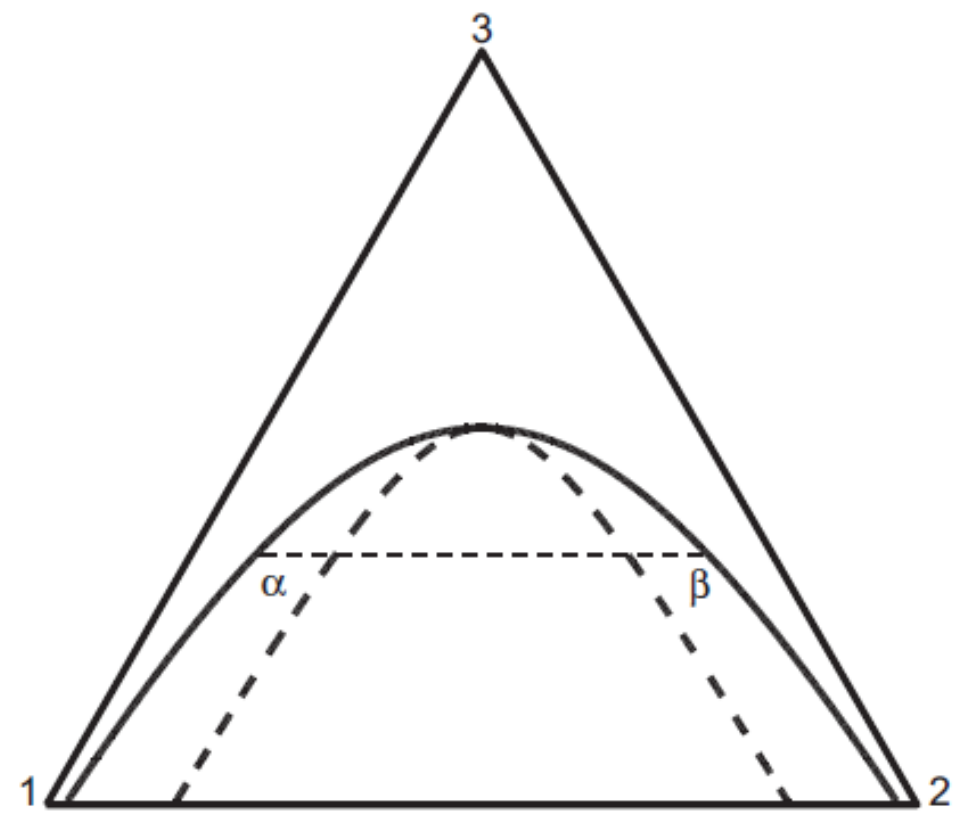

Figure 1: Phase diagram for $\Psi_{12}=4, \Psi_{23}=\Psi_{31}=0$. 


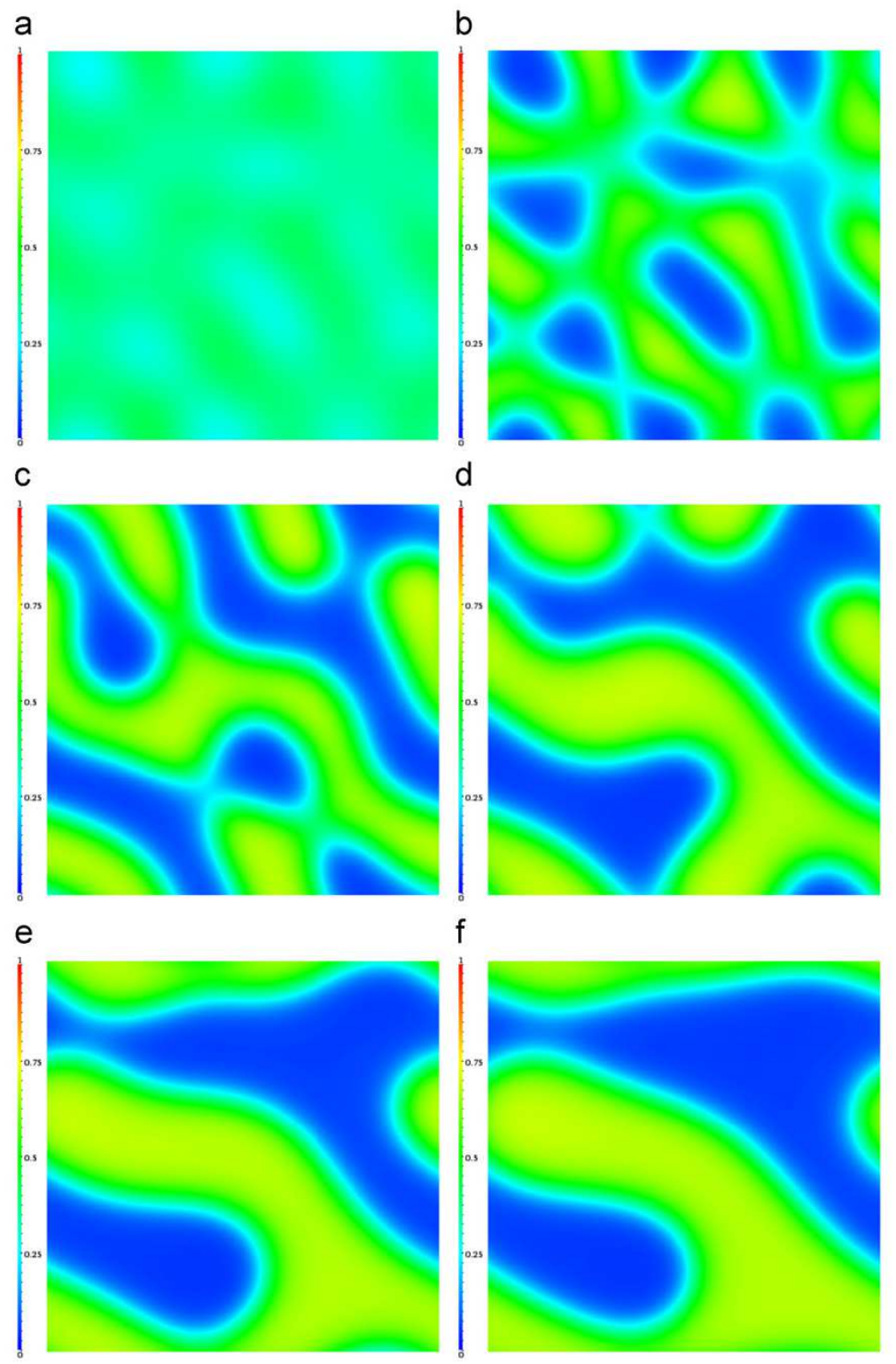

Figure 2: Evolution of $x_{1}$ for two-phase mixture: (a) $t=120$, (b) $t=160$, (c) $t=320$, (d) $t=480$, (e) $t=640$, and (f) $t=800$. 


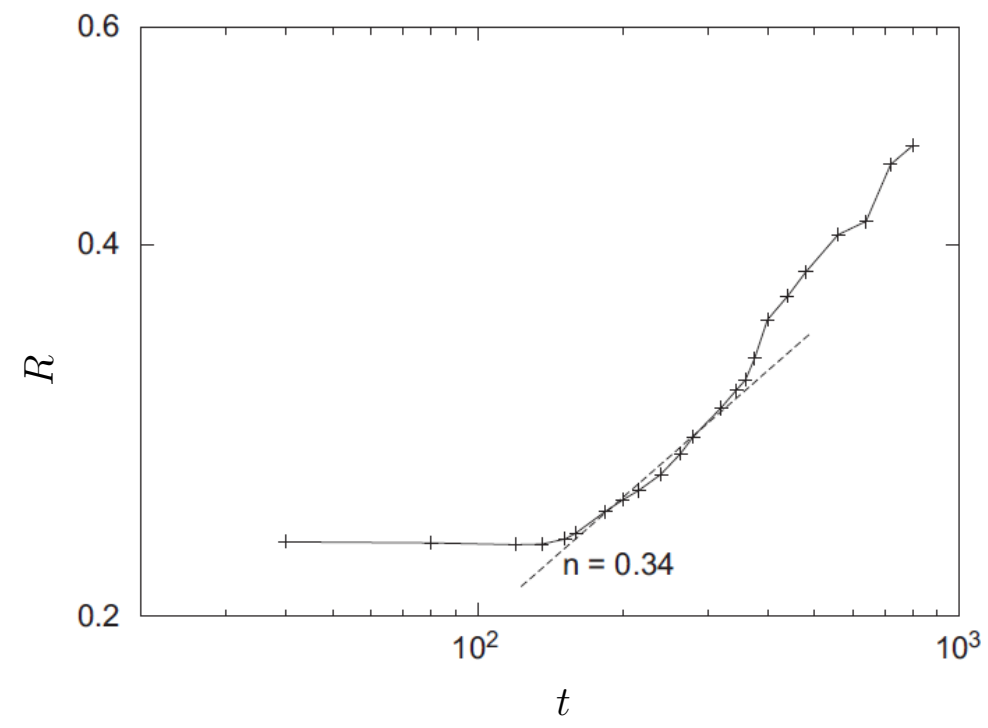

Figure 3: Growth of domain size in two-phase case.

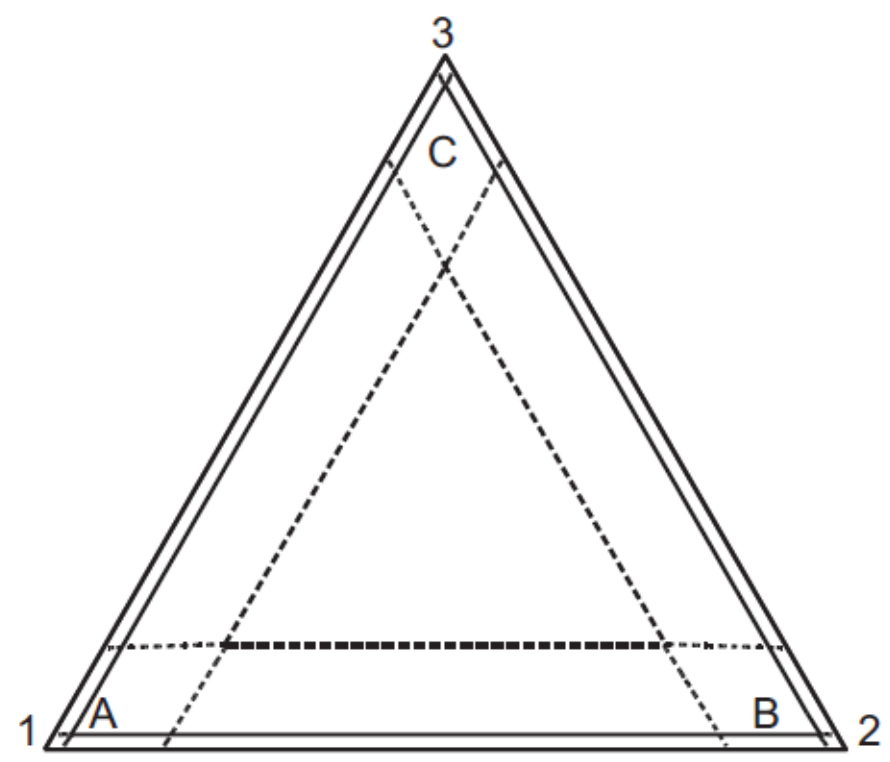

Figure 4: Phase diagram for $\Psi_{12}=\Psi_{23}=\Psi_{31}=4$. 


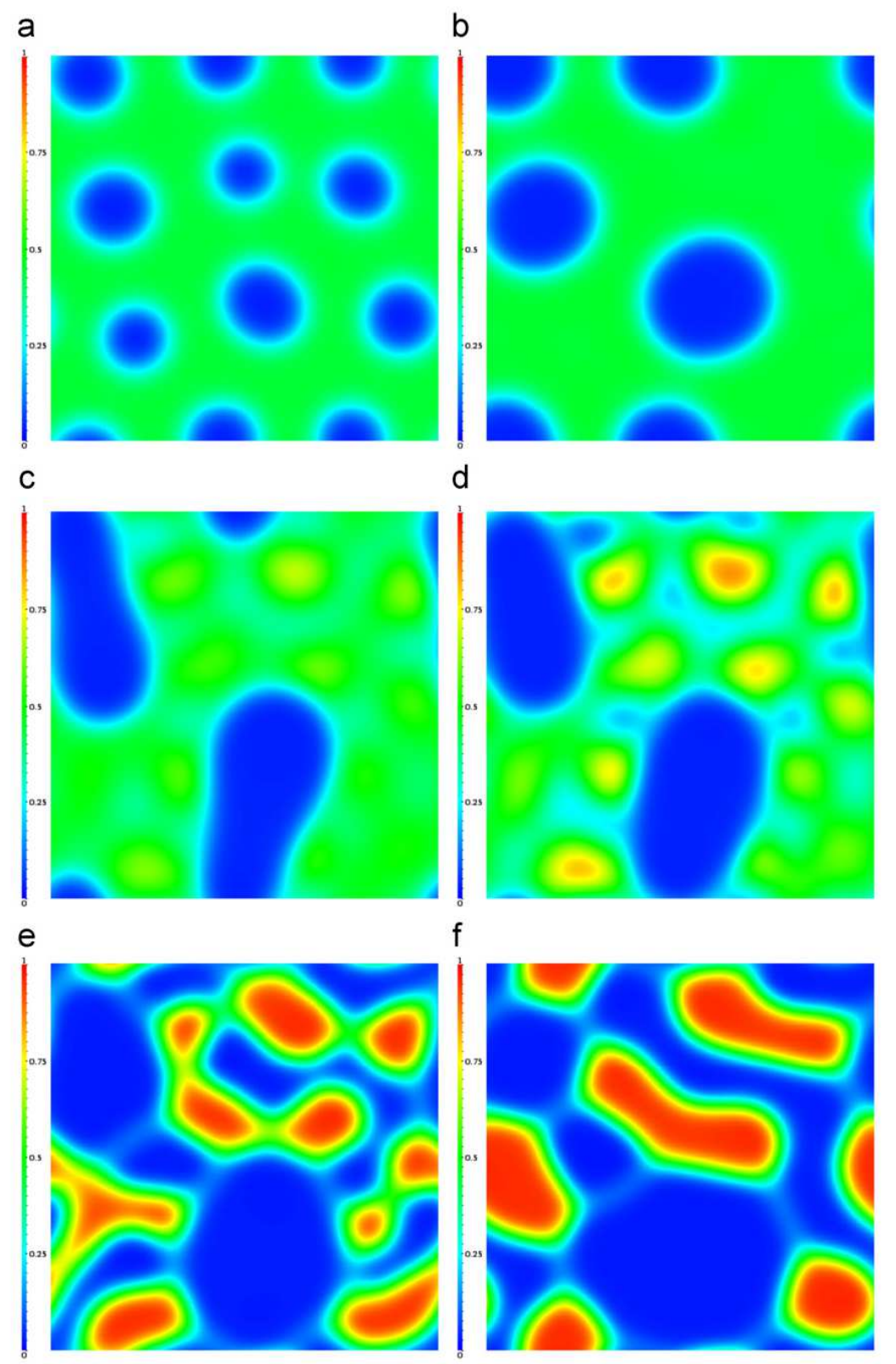

Figure 5: Evolution of $x_{1}$ for three-phase mixture: (a) $t=24$, (b) $t=72$, (c) $t=120$, (d) $t=136$, (e) $t=200$, and (f) $t=400$. 


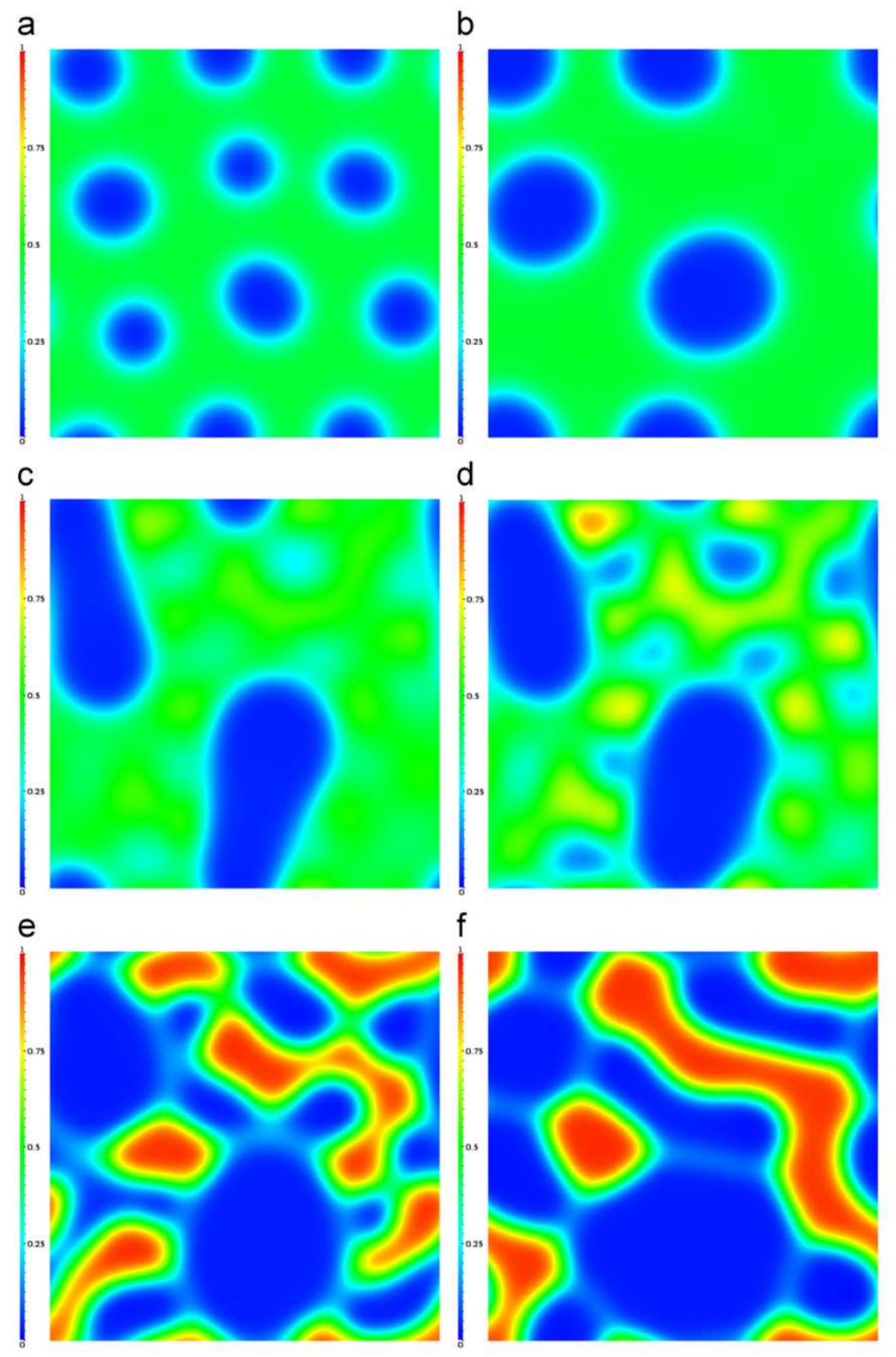

Figure 6: Evolution of $x_{2}$ for three-phase mixture: (a) $t=24$, (b) $t=72$, (c) $t=120$, (d) $t=136$, (e) $t=200$, and (f) $t=400$. 

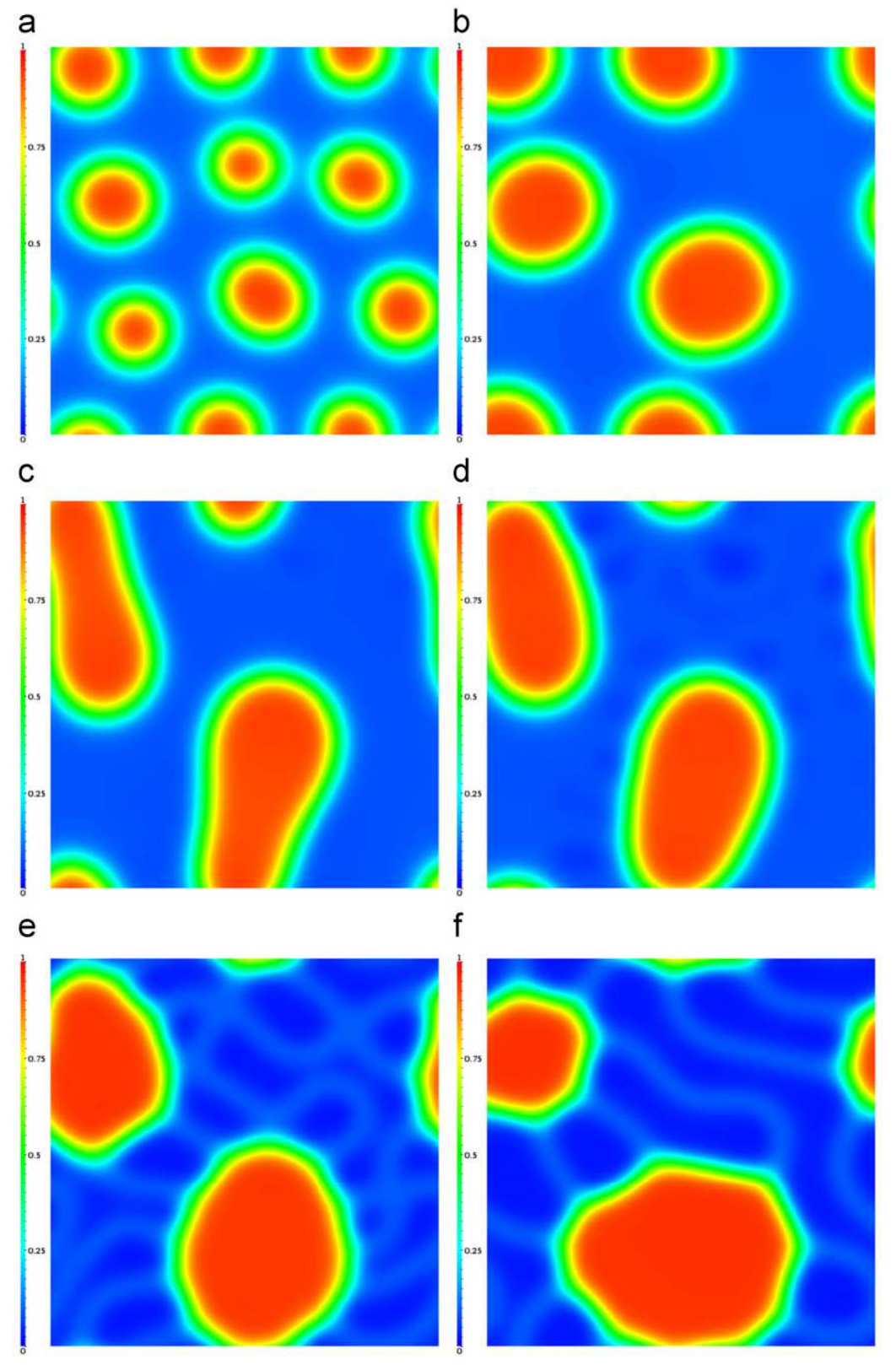

Figure 7: Evolution of $x_{3}$ for three-phase mixture: (a) $t=24$, (b) $t=72$, (c) $t=120$, (d) $t=136$, (e) $t=200$, and (f) $t=400$. 


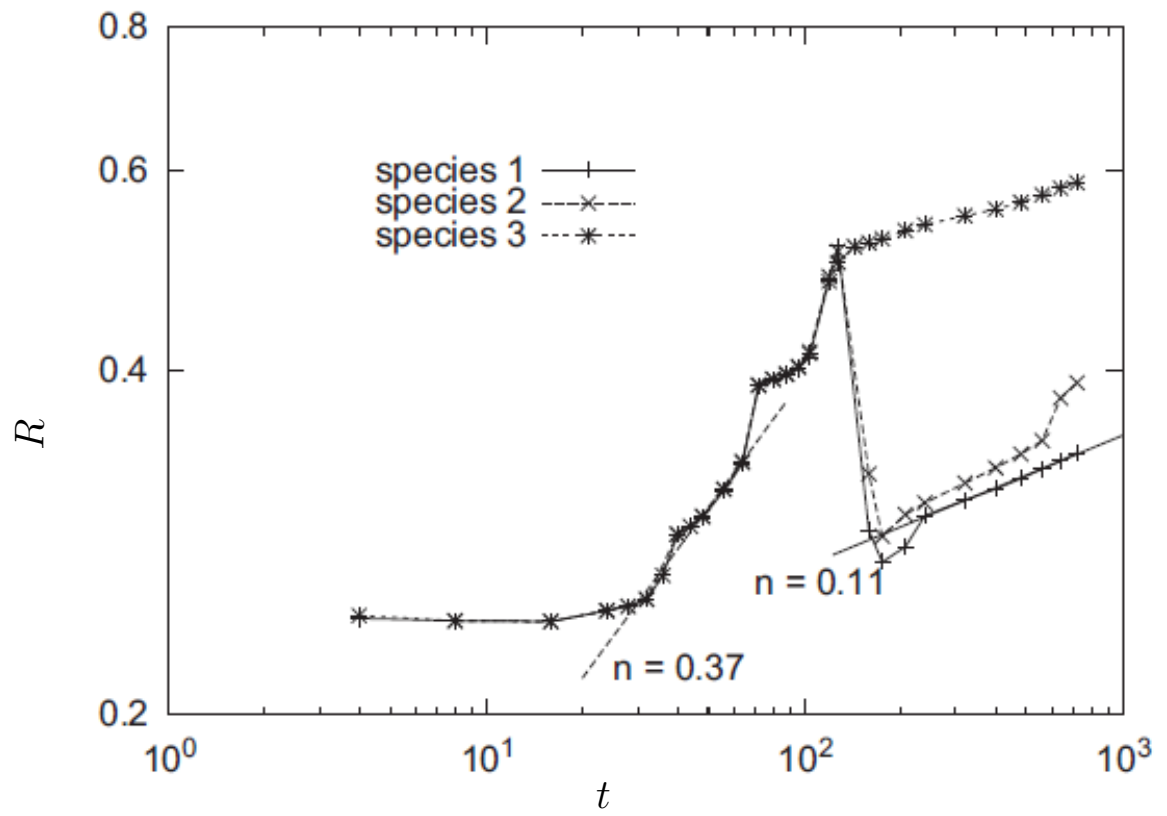

Figure 8: Growth of domain size in three-phase case. 